\title{
ASSESSING THE HABITAT SUITABILITY OF TWO DIFFERENT ARTIFICIAL WETLAND HABITATS USING AVIAN COMMUNITY STRUCTURES
}

\author{
Zakaria, M. and M.N. Rajpar \\ Department of Recreation and Ecotourism, Faculty of Forestry, \\ Universiti Putra Malaysia, 43400 UPM Serdang, Selangor Darul Ehsan, Malaysia
}

Received 2014-01-23; Revised 2014-02-12; Accepted 2014-05-24

\begin{abstract}
Artificial wetlands have become highly important habitat for a variety of bird species particularly waterbirds due to loss and degradation of natural wetlands. Avian community structures in two artificial wetland habitats i.e., man-made marsh and lake were investigated to identify the suitability of the habitats for avian species, habitat preference and food resources. Distance sampling point count method detected 35272 bird individuals of 98 species from July to November 2010. Ninty four bird species were recorded from man-made marsh and thirty one species from the lake area. Acridotheres tristis $(6.34 \%$ of all detections) was the most dominant bird species of man-made marsh and Passer montanus (0.41\%) was the most dominant bird species of lake habitat. On the contrarily, Microhierax fringillarius and Turdoides earlei (each $0.01 \%$ ) were the rarest species of man-made marsh and Dinopium javanense $(0.02 \%)$ was the rarest species of the lake habitat. The bird relative abundance of man-made marsh and lake habitats was significantly different $\left(\mathrm{F}_{1}, 194=50.3, \mathrm{p}<0.05\right)$. Community analysis indicated that bird species of man-made marsh were more diverse i.e., Shannon's index $\left(\mathrm{N}_{1}=3.92\right)$ and rich i.e., Margalef's index $\left(\mathrm{R}_{1}=8.93\right)$ than the lake habitat. Insectivore (marsh; $21.13 \%$ and lake area; $1.21 \%$ ) was the most dominant guild in manmade marsh and lake area. In contrast, Piscivore $(0.14 \%)$ was the rarest guild comprised of only one species in marsh habitat. The results of this study revealed that the man-made marsh was more capable to attract a higher number of bird species and diversity than the lake habitat.
\end{abstract}

Keywords: Birds, Community Structure, Diversity, Habitat, Marsh, Lake and Feeding Guild

\section{INTRODUCTION}

Worldwide, more than $50 \%$ of natural wetland areas have been lost due to human activities which had adversely affected hydro system, plant growth and avian communities that depend on wetland habitats directly and indirectly for various activities (Fraser and Keddy, 2005; Coleman et al., 2008; Mitsch, 2010). Due to loss and degradation of natural wetlands, man-made marshes and lake areas had become ecological important habitats for many avian species (White, 2003; Yaukey, 2011; Zakaria and Rajpar, 2013).

Artificial wetland is a type of aquatic habitat constructed manually and planted with herbaceous vegetation to trap the sediements from surrounded catchement areas and also to retain water for drinking or irrigation purpose (Vymazal, 2010). As the wastewater flows from catchment area through the length of the aquatic habitat, suspended solids and matter settle out and bottom sediments trapped toxic contaminants and pollutants through microbial interaction (Kirby, 2002). Vegetation plays a vital role in hydrological processes, reducing soil erosion, filter and trap sedimentation from catchment inflows, reduced turbidity that had contributed to high water quality and aquatic life (Loaiza, 2008; Li and Yang, 2009; Ekert, 2009).

Man-made marshes are dominated by extensive growths of herbaceous plants (such as grasses, sedges, Corresponding Author: Zakaria, M., Department of Recreation and Ecotourism, Faculty of Forestry, Universiti Putra Malaysia, 43400 UPM Serdang, Selangor Darul Ehsan, Malaysia 
reeds and cattails) rooted in and usually protruding from, shallow water that stands above the ground surface to harbor wide array of birds, amphibians, reptiles, fishes and aquatic invertebrates (Ma et al., 2010; Haber, 2011; Ramírez et al., 2012). These habitats can promote sustainable use of local resources and are environmental friendly (Kadlec et al., 2000; Sim, 2003). They have potential to provide alternative habitat instead of natural habitat for avian species and can mitigate the adverse effects of wetland loss and degradation (Connor and Gabor, 2006; Sim et al., 2008; Ma et al., 2010; Rajpar and Zakaria, 2013).

Birds are the bioindicators of aquatic habitats such as man-made marshes and lake areas. Their occurrence or absence of bird species may indicate the habitat characteristics and productivity of the particular habitat (Noble et al., 2008; Larsen et al., 2010; Rajpar and Zakaria, 2011a; BLI, 2013). Investigating avian community structures (i.e., abundance, diversity, feeding guilds and habitat preference) is a crucial tool to determine the habitat suitability and to detect the changes within particular habitat during specific period (DeSante et al., 2005). Information related to avian community structures in artificial wetlands such as man-made marshes and lake areas of Malaysia is still lacking. To date, no detailed study has been in such types of artificial wetland habitats. The aim of this research was to assess the habitat suitability of artificial wetland habitats i.e., man-made marsh and lake area to understand the importance of each habitat as alternative habitat to natural one for future conservation and management planning.

\section{MATERIALS AND METHODS}

\subsection{Study Area}

Man-made marsh and lake habitat is situated about 26 $\mathrm{km}$ south of Kuala Lumpur within the quadrant of $2^{\circ} 57^{\prime}$ 43" latitude and $101^{\circ} 41^{\prime} 47^{\prime \prime}$ longitude (Fig. 1). Manmade marsh occupy an area of 200 ha and lake cover 400 ha. This man-made marsh and lake was constructed in 1997 to trap the pollutants derived from upstream sources, to control the inflow of flood water from catchment areas of the Chua and Bisa Rivers, to retain water for domestic use and increased the aesthetic value of the Putrajaya administrative city. These areas straddle the water from Chua River, Bisa River and three tributaries. This manmade marsh and lake is still in an early phase of succession and is far less complex than the natural wetland (Ismail et al., 2012). These artificial wetlands (man-made marsh and lake) is dominated by a wide range of aquatic plants (e.g., spike rush-Eleocharis varigata, water chestnut-E. dulcis, blue sedge-Lepironia articulate, water
hyacinth-Eichhornia crassipes and duckweed-Lemna sp.), fruiting trees (e.g., brush cherries-Eugenia sp., weeping fig-Ficus benamina and golden ficus- $F$. microcarpa), flowering trees and bushes (e.g., flame tree-Delonix regia, beach hibiscus-Hibiscus tiliaceus and gelam-Melaleuca cajuputi) and shrubs (e.g., fish-tailed palm-Caryota mitis, island lychee-Pometia pinnata and simpoh ayer-Dillenia suffruticosa) (Rajpar and Zakaria, 2013).

\subsection{Bird Surveys}

Bird were surveyed employing the distance sampling point count technique to determine the aian community structures i.e., relative abundance, diversity, feeding guilds and habitat preference from July to November, 2010. A total of 89 point count stations (i.e., 45 point count stations in man-made marsh and 43 point count stations in the lake habitat) were established at $300 \mathrm{~m}$ intervals along the wetland edges. This distance was selected to obtain more reliable data and avoid the detection of the same bird individual at more than one station. The birds were surveyed in each month for eight days and each point count station was surveyed for 10 min by walk along trails set along the dikes. During each survey, all bird species and individuals seen or heard were recorded. The flushed birds with known original positions were also recorded and included in the analysis. The distances from bird individual to observer was recorded using visual estimation for each bird individual within the range of $100 \mathrm{~m}$. The surveys were conducted by a single observer between 0730 and 1100 $\mathrm{h}$. This period of time is an appropriate, as most birds are active early in the morning. The survey methodology was based on (Buckland et al., 2004; Nadeau et al., 2008; Aynalem and Bekele, 2008; Zakaria et al., 2009).

\subsection{Data Analysis}

\subsubsection{Relative Abundance (\%)}

Relative abundance refers to the number of bird individual detections of a particular species as percent of the total detected in man-made marsh and lake habitats. We estimated relative abundances for each species using average detection values obtained by dividing the total number of a species detected at man-made marsh and lake habitats. The relative abundance (\%) of bird species was estimated using the following expression:

$$
\text { Relative abundance }=\mathrm{n} / \mathrm{N} \times 100
$$

Where:

$\mathrm{n}=$ Number of a particular detected bird species and

$\mathrm{N}=$ Total detected bird individuals in man-made marsh and lake habitats 


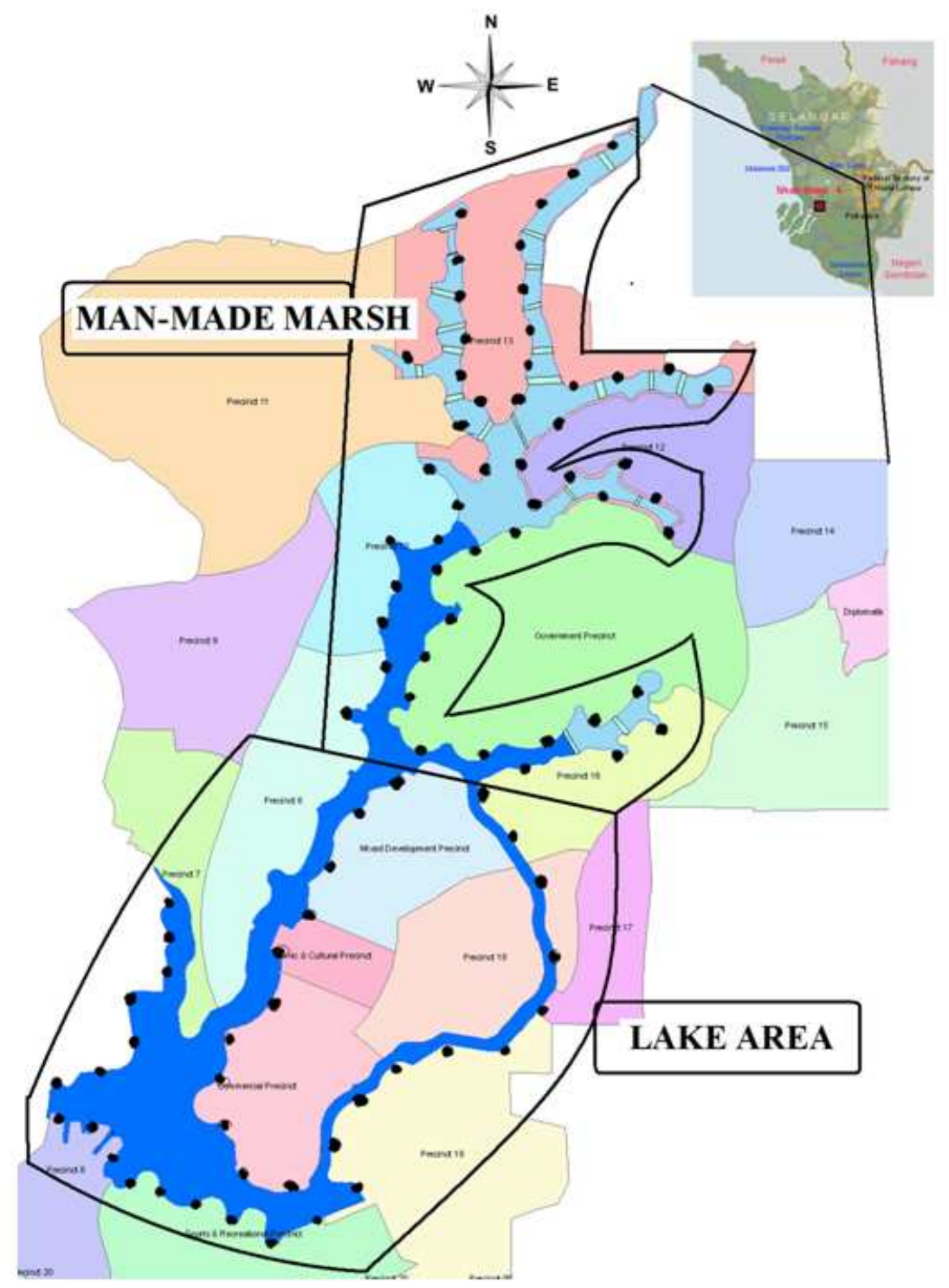

Fig. 1. Map location of the distance sampling point count stations in man-made marsh and lake area at Putrajaya Wetland, Peninsular Malaysia

\subsubsection{Bird Diversity Indices}

Avian species diversity, species richness and species evenness in man-made marsh and lake habitats were analyzed using Community Analysis Package Software (CAP, Version 4.0) (Henderson and Seaby, 2007).

Shannon-weiner diversity index:

$$
H^{\prime}=\sum_{i=1}^{R} p_{i} \ln p_{i}
$$

Where:

$\mathrm{S}=$ Total number of individual

$\mathrm{P}_{\mathrm{i}}=\mathrm{N}_{\mathrm{i}} / \mathrm{N}=$ proportion of individuals of the total sample belonging to ${ }_{i}$ th species

$\mathrm{Ni}=$ number of individuals $(\mathrm{N})$ belonging to the ${ }_{\mathrm{i}}$ th species and

$\mathrm{N}=$ total number of individuals

Margalef's richness index was calculated using the following equation: 


$$
\mathrm{R}_{1}=(\mathrm{S}-1) / \ln (\mathrm{n})
$$

Where:

$\mathrm{S}=$ Number of taxa and

$\mathrm{n}=$ Number of individuals

McIntosh evenness index E was calculated using the following equation:

$$
\operatorname{Mc} E=\left[N-\sqrt{ }\left(\sum n_{i}^{2}\right)\right] /[N-(N / \sqrt{S})]
$$

Where:

Mc E = McIntosh evenness index

$\mathrm{n}_{\mathrm{i}} \quad=$ Number of individuals belonging to i species

$\mathrm{S} \quad=$ Total number of species and

$\mathrm{N}=$ Total number of individuals

\subsubsection{Feeding Guild}

Bird species was grouped into functional guilds that may reflect the exploitation of same food resources and foraging technique in a similar way in a particular habitat. Thus, we assigned all recorded bird species into eight major feeding guilds such as Insectivore, Frugivore/Insectivore, Granivore, Omnivore, Piscivore/Carnivore/Insectivore, Granivore/Insectivore, Nectarivore/Insectivore, Frugivore, Insectivore/Nectarivore/Frugivore, Piscivore/Insectivore, Carnivore/Insectivore, Piscivore/Carnivore, Carnivore and Piscivore based on their major food resources, foraging behavior and habitat selection (Zakaria et al., 2009; BLI, 2013).

\subsubsection{Significant Difference among Different Aged Post-Harvested Forest Habitats}

A One-Way Analysis of Variance (ANOVA) and Tukey's (HSD) test (Analytical Software, version 8.1) by McGraw-Hill (2008) was conducted in order to investigate the difference in bird richness and habitat characteristics between primary and logged hill dipterocarp tropical rainforest.

\section{RESULTS}

From July to November, 2010, a total of 35272 bird observations representing 98 species and 38 families was recorded in man-made marsh and lake area. Twenty seven species were recorded in both habitats. Sixty seven bird species were recorded only in the man-made marsh which were absent in the lake habitat. Likewise, four species were only detected in the lake area and were absent in man-made marsh habitat.

\subsection{Relative Abundance of Birds in Man-Made Marsh Habitat}

A total of 33,464 bird detections, that is $94.87 \%$ of all detections were recorded in the man-made marsh area. The bird belongs to 94 species and 36 families. Three species, Acridotheres tristis-Common Myna (6.34\% of all detections), Pycnonotus goiavier-Yellow-vented Bulbul $(5.85 \%)$ and Acridotheres fuscus-Jungle Myna $(4.68 \%)$ indicated the highest relative abundance. In contrast, two bird species, Microhierax fringillariusBlack-thighed Falconet and Turdoides earlei-Striated Babbler $(0.01 \%$ each) were the least abundant species in the marsh area (Table 1).

\subsection{Relative Abundance of Birds in Lake Habitat}

A total of 1,808 bird individuals $(5.13 \%$ of all detections) was recorded in the lake area which included 31 species and 22 families. Passer montanus-Eurasian Tree-Sparrow ( $0.4 \%$ of all detections), Acridotheres tristis-Common Myna (0.35\%), Pycnonotus goiavierYellow-vented Bulbul $(0.33 \%)$ were the three most abundant birds in the lake area. Whereas, two bird species, Artamas leucorynchus-White-breasted Wood Swallow $(0.03 \%)$ and Dinopium javanense-Common Flameback $(0.02 \%)$ were the least common in the lake area (Table 1).

\subsection{Comparison of Bird Species Diversity in Man-made Marsh and Lake Habitats}

Results indicated that bird mean relative abundance of man-made marsh is significantly different from lake area $\left(\mathrm{F}_{1},{ }_{194}=50.3, \mathrm{p}<0.05\right.$; Table 2). Community analysis indicated that higher species diversity, i.e., Shannon's index; $N_{1}=3.92$ and species richness, i.e., Margalef's index; $\mathrm{R}_{1}=8.93$ occurred in the man-marsh area while higher species evenness, i.e., Pielou's J index $\mathrm{E}=0.95$ was recorded in the lake area (Table 3).

The significant difference of bird diversity (i.e., species diversity, richness and evenness) for man-made marsh and lake area was determined employing ANOVA and Tukey's (HSD) test. Results showed that bird diversity of man-made marsh is not significantly different from the lake area $\left(\mathrm{F}_{1},{ }_{4}=0.53, \mathrm{p}>0.05\right.$; Table 4).

\subsection{Comparison of Feeding Guilds between Man-Made Marsh and Lake Habitats}

Ninety eight bird species were categorized into fourteen major feeding guilds based on detected foraging behaviour and food consumption at foraging sites. The feeding guild structure indicated that Insectivore (marsh; $21.13 \% ; 22$ bird species and lake area; $1.21 \%$; 9 species) was the most dominant guild in both habitats. 
Zakaria, M. and M.N. Rajpar / American Journal of Applied Sciences 11 (8): 1321-1331, 2014

Table 1. List of bird species with relative abundance recorded in man-made marsh and lake area at Putrajaya, Peninsular Malaysia

\begin{tabular}{|c|c|c|c|c|c|c|}
\hline \multirow[b]{2}{*}{ Family name } & \multirow[b]{2}{*}{ Scientific name } & \multirow[b]{2}{*}{ Common name } & \multicolumn{2}{|c|}{ Man-made Marsh } & \multicolumn{2}{|l|}{ Lake Area } \\
\hline & & & Observation & $\begin{array}{l}\% \text { of all } \\
\text { detections }\end{array}$ & Observation & $\begin{array}{l}\% \text { of all } \\
\text { detections }\end{array}$ \\
\hline Sturnidae & Acridotheres tristis & Common Myna & 2237 & 6.34 & 124 & 0.35 \\
\hline Pycnonotidae & Pycnonotus goiavier & Yellow-vented Bulbul & 2065 & 5.85 & 115 & 0.33 \\
\hline Sturnidae & Acridotheres fuscus & Jungle Myna & 1650 & 4.68 & 102 & 0.29 \\
\hline Columbidae & Treron vernans & Pink-necked Green Pigeon & 1608 & 4.56 & 0 & 0.00 \\
\hline Columbidae & Geopelia striata & Peaceful Dove & 1493 & 4.23 & 102 & 0.29 \\
\hline Passeridae & Passer montanus & Eurasian Tree Sparrow & 1342 & 3.80 & 145 & 0.41 \\
\hline Muscicapidae & Copsychus saularis & Oriental Magpie Robin & 1144 & 3.24 & 92 & 0.26 \\
\hline Rallidae & Amaurornis phoenicurus & White-breasted Waterhen & 926 & 2.63 & 58 & 0.16 \\
\hline Columbidae & Streptopelia chinensis & Spotted Dove & 916 & 2.60 & 63 & 0.18 \\
\hline Hirundinidae & Hirundo tahitica & House Swallow & 882 & 2.50 & 0 & 0.00 \\
\hline Oriolidae & Oriolus chinensis & Black-naped Oriole & 839 & 2.39 & 85 & 0.24 \\
\hline Estrildidae & Lonchura punctulata & Scaly-breasted Munia & 822 & 2.33 & 61 & 0.17 \\
\hline Aegithinidae & Aegithina tiphia & Common Iora & 776 & 2.20 & 72 & 0.20 \\
\hline Sturnidae & Aplonis panayensis & Asian Glossy Starling & 774 & 2.19 & 69 & 0.19 \\
\hline Rhipiduridae & Rhipidura javanica & Pied Fantail & 682 & 1.93 & 43 & 0.12 \\
\hline Ploceidae & Ploceus philippinus & Baya Weaver & 655 & 1.86 & 69 & 0.19 \\
\hline Sturnidae & Hirundo rustica & Barn Swallow & 649 & 1.84 & 0 & 0.00 \\
\hline Nectariniidae & Arachnothera longirostra & Little Spiderhunter & 599 & 1.70 & 69 & 0.19 \\
\hline Estrildidae & Lonchura maja & White-headed Munia & 573 & 1.62 & 0 & 0.00 \\
\hline Nectariniidae & Cinnyris jugularis & Olive-backed Sunbird & 568 & 1.61 & 0 & 0.00 \\
\hline Alcidinidae & Halcyon smyrnensis & White-throated Kingfisher & 525 & 1.49 & 37 & 0.10 \\
\hline Ardeidae & Egretta garzetta & Little Egret & 513 & 1.45 & 0 & 0.00 \\
\hline Nectariniidae & Anthreptes malacensis & Brown-throated Sunbird & 489 & 1.39 & 35 & 0.10 \\
\hline Cisticolidae & Orthotomus sutorius & Common Tailorbird & 470 & 1.33 & 74 & 0.21 \\
\hline Ardeidae & Ardea purpurea & Purple Heron & 463 & 1.31 & 0 & 0.00 \\
\hline Meropidae & Merops leschenaulti & Chestnut-headed Bee-Eater & 458 & 1.30 & 0 & 0.00 \\
\hline Columbidae & Columba livia & Rock Pigeon & 429 & 1.22 & 0 & 0.00 \\
\hline Ardeidae & Bubulcus coromandus & Eastern Cattle Egret & 423 & 1.20 & 0 & 0.00 \\
\hline Alcidinidae & Alcedo atthis & Common Kingfisher & 420 & 1.19 & 0 & 0.00 \\
\hline Meropidae & Merops philippinus & Blue-tailed Bee-Eater & 405 & 1.15 & 0 & 0.00 \\
\hline Ardeidae & Nycticorax nycticorax & Black-crowned Night-Heron & 388 & 1.10 & 0 & 0.00 \\
\hline Motacillidae & Anthus richardi & Richard's Pipit & 361 & 1.02 & 43 & 0.12 \\
\hline Cuculidae & Cypsiurus balasiensis & Asian Palm Swift & 353 & 1.00 & 0 & 0.00 \\
\hline Nectariniidae & Anthreptes simplex & Plain Sunbird & 321 & 0.91 & 0 & 0.00 \\
\hline Sturnidae & Acridotheres grandis & White-vented Myna & 318 & 0.90 & 26 & 0.07 \\
\hline Cisticolidae & Prinia flaviventris & Yellow-bellied Prinia & 303 & 0.86 & 16 & 0.05 \\
\hline Ardeidae & Butorides striata & Little Heron & 302 & 0.86 & 39 & 0.11 \\
\hline Estrildidae & Lonchura ferruginosa & White-capped Munia & 296 & 0.84 & 0 & 0.00 \\
\hline Ardeidae & Ixobrychus sinensis & Yellow Bittern & 270 & 0.77 & 0 & 0.00 \\
\hline Rallidae & Porphyrio porphyrio & Purple Swamphen & 268 & 0.76 & 0 & 0.00 \\
\hline Cisticolidae & Orthotomus atrogularis & Dark-necked Tailorbird & 266 & 0.75 & 0 & 0.00 \\
\hline Pycnonotidae & Pycnonotus atriceps & Black-headed Bulbul & 237 & 0.67 & 0 & 0.00 \\
\hline Ciconiidae & Mycteria leucocephala & Painted Stork & 224 & 0.64 & 0 & 0.00 \\
\hline Nectariniidae & Arachnothera affinis & Grey-breasted spiderhunter & 205 & 0.58 & 0 & 0.00 \\
\hline Columbidae & Treron olax & Little Green-Pigeon & 196 & 0.56 & 0 & 0.00 \\
\hline Alcidinidae & Halcyon pileata & Black-capped Kingfisher & 173 & 0.49 & 0 & 0.00 \\
\hline Cisticolidae & Orthotomus ruficeps & Ashy Tailorbird & 169 & 0.48 & 61 & 0.17 \\
\hline Alcidinidae & Todiramphus chloris & Collared Kingfisher & 160 & 0.46 & 0 & 0.00 \\
\hline Dicaeidae & Dicaeum maculatus & Yellow-breasted Flowerpecker & 158 & 0.45 & 0 & 0.00 \\
\hline Psittacidae & Loriculus galgulus & Blue-crowned hanging parrot & 148 & 0.42 & 0 & 0.00 \\
\hline Ardeidae & Ixobrychus cinnamomeus & Cinnamon Bittern & 144 & 0.41 & 0 & 0.00 \\
\hline Laniidae & Lanius cristatus & Brown Shrike & 134 & 0.38 & 0 & 0.00 \\
\hline Picidae & Meiglyptes tristis & Buff-rumped woodpecker & 114 & 0.33 & 0 & 0.00 \\
\hline Timaliidae & Macronus gularis & Pin-striped tit-babbler & 110 & 0.32 & 0 & 0.00 \\
\hline
\end{tabular}


Zakaria, M. and M.N. Rajpar / American Journal of Applied Sciences 11 (8): 1321-1331, 2014

Table 1. Continue

\begin{tabular}{|c|c|c|c|c|c|c|}
\hline Pycnonotidae & Pycnonotus simplex & Cream-vented Bulbul & 108 & 0.31 & 0 & 0.00 \\
\hline Corvidae & Corvus splendens & House Crow & 108 & 0.31 & 48 & 0.14 \\
\hline Ardeidae & Ardea cinerea & Grey Heron & 107 & 0.30 & 0 & 0.00 \\
\hline Ardeidae & Ardea alba & Great Egret & 102 & 0.29 & 0 & 0.00 \\
\hline Campephagidae & Lalage nigra & Pied Triller & 85 & 0.25 & 0 & 0.00 \\
\hline Laniidae & Lanius tigrinus & Tiger Shrike & 84 & 0.24 & 0 & 0.00 \\
\hline Laniidae & Hemipus hirundinaceus & Black-winged Flycatcher Shrike & 0 & 0.00 & 17 & 0.05 \\
\hline Turnicidae & Hemipus picatus & Bar-winged Flycatcher-Shrike & 81 & 0.23 & 0 & 0.00 \\
\hline Cuculidae & Centropus bengalensis & Lesser Coucal & 81 & 0.23 & 0 & 0.00 \\
\hline Hirundinidae & Turnix suscitator & Barred Buttonquail & 79 & 0.22 & 27 & 0.08 \\
\hline Dicaeidae & Dicaeum trigonostigma & Orange-bellied Flowerpecker & 79 & 0.22 & 0 & 0.00 \\
\hline Nectariniidae & Anthreptes rhodolaema & Red-throated Sunbird & 79 & 0.22 & 0 & 0.00 \\
\hline Muscicapidae & Ficedula zanthopygia & Yellow-rumped Flycatcher & 79 & 0.22 & 0 & 0.00 \\
\hline Charadriidae & Vanellus indicus & Red-wattled Lapwing & 74 & 0.21 & 0 & 0.00 \\
\hline Picidae & Micropternus brachyurus & Rufous Woodpecker & 74 & 0.21 & 0 & 0.00 \\
\hline Cuculidae & Rhinortha chlorophaeus & Raffle's Malkoha & 71 & 0.20 & 0 & 0.00 \\
\hline Columbidae & Chalcophaps indica & Emerald Dove & 70 & 0.19 & 0 & 0.00 \\
\hline Sturnidae & Gracupica contra & Asian Pied Starling & 58 & 0.16 & 0 & 0.00 \\
\hline Ardeidae & Ardeola speciosa & Javan Pond Heron & 54 & 0.15 & 0 & 0.00 \\
\hline Pelecanidae & Pelecanus onocrotalus & Great White Pelican & 50 & 0.14 & 0 & 0.00 \\
\hline Nectariniidae & Chalcoparia singalensis & Ruby-cheeked Sunbird & 49 & 0.13 & 0 & 0.00 \\
\hline Falconidae & Spilornis cheela & Crested Serpent Eagle & 44 & 0.12 & 0 & 0.00 \\
\hline Phasianidae & Gallus gallus & Red Junglefowl & 41 & 0.11 & 0 & 0.00 \\
\hline Timaliidae & Malacocincla abbotti & Abbott's Babbler & 40 & 0.11 & 0 & 0.00 \\
\hline Picidae & Dinopium javanense & Common Flameback & 39 & 0.11 & 8 & 0.02 \\
\hline Cuculidae & Cacomantis sepulcralis & Rusty-breasted Cuckoo & 38 & 0.10 & 0 & 0.00 \\
\hline Dicaeidae & Dicaeum thoracicus & Scarlet-breasted Flowerpecker & 35 & 0.09 & 0 & 0.00 \\
\hline Alcidinidae & Alcedo meninting & Blue-eared Kingfisher & 30 & 0.08 & 0 & 0.00 \\
\hline Timaliidae & Zosterops palpebrosus & Oriental White Eye & 27 & 0.07 & 0 & 0.00 \\
\hline Dicaeidae & Dicaeum cruentatum & Scarlet-backed Flowerpecker & 26 & 0.07 & 0 & 0.00 \\
\hline Nectariniidae & Arachnothera flavigaster & Spectacled Spiderhunter & 24 & 0.06 & 0 & 0.00 \\
\hline Nectariniidae & Aethopyga nipalensis & Green-tailed Sunbird & 21 & 0.06 & 0 & 0.00 \\
\hline Falconidae & Haliaeetus leucogaster & White-bellied Sea-Eagle & 20 & 0.05 & 0 & 0.00 \\
\hline Muscicapidae & Muscicapa dauurica & Asian Brown Flycatcher & 19 & 0.05 & 0 & 0.00 \\
\hline Megalaimidae & Megalaima haemacephala & Coppersmith Barbet & 18 & 0.05 & 0 & 0.00 \\
\hline Cuculidae & Cuculus micropterus & Indian Cuckoo & 18 & 0.05 & 0 & 0.00 \\
\hline Coraciidae & Eurystomus orientalis & Dollar Bird & 17 & 0.04 & 0 & 0.00 \\
\hline Scolopacidae & Actitis hypoleucos & Common Sandpiper & 8 & 0.02 & 49 & 0.14 \\
\hline Muscicapidae & Ficedula mugimaki & Mugimaki Flycatcher & 6 & 0.02 & 0 & 0.00 \\
\hline Falconidae & Microhierax fringillarius & Black-thighed Falconet & 4 & 0.01 & 0 & 0.00 \\
\hline Timaliidae & Turdoides earlei & Striated Babbler & 4 & 0.01 & 0 & 0.00 \\
\hline Cuclidae & Eudynamys scolpaceus & Common Asian Koel & 0 & 0.00 & 24 & 0.07 \\
\hline Anatidae & Anas crecca & Eurasian Teal & 0 & 0.00 & 24 & 0.07 \\
\hline Artamidae & Artamas leucorynchus & White-breasted Wood Swallow & 0 & 0.00 & 11 & 0.03 \\
\hline Total & & & 33,464 & & 1808 & \\
\hline
\end{tabular}

Table 2. Comparison of relative abundance of bird species in man-made marsh and lake area

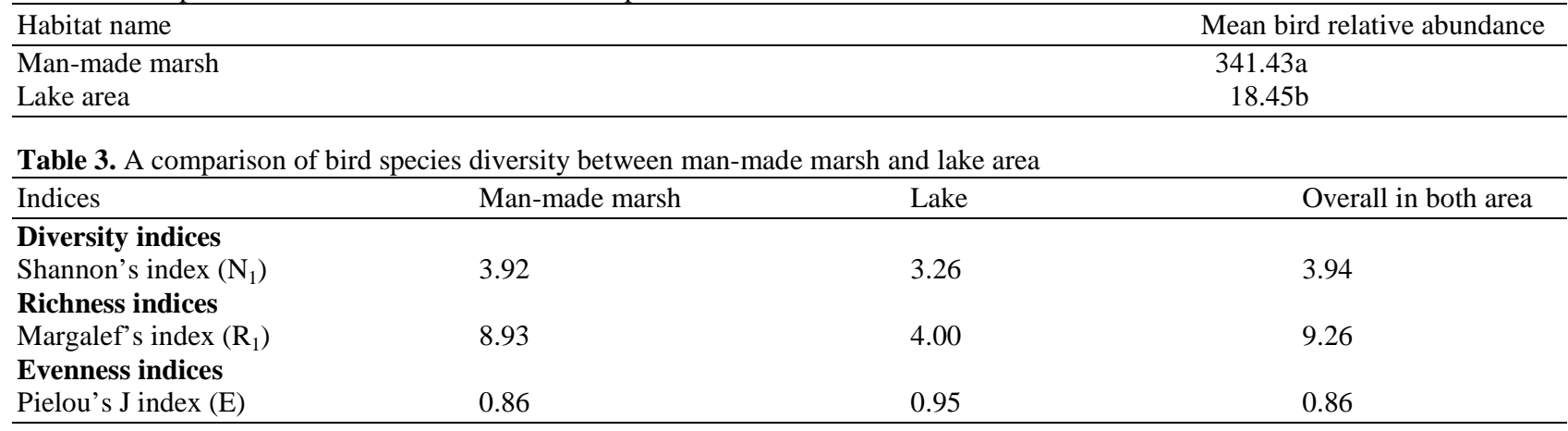


Table 4. Comparison of bird diversity in man-made marsh and lake area

\begin{tabular}{lc}
\hline Habitat name & Mean diversity \\
\hline Man-made marsh & $4.57 \mathrm{a}$ \\
Lake area & $2.76 \mathrm{a}$ \\
\hline
\end{tabular}

Table 5. Comparison of feeding guilds in marsh and lake areas

\begin{tabular}{|c|c|c|c|c|}
\hline \multirow[b]{2}{*}{ Name of guild } & \multicolumn{2}{|l|}{ Man-made marsh area } & \multicolumn{2}{|l|}{ Lake area } \\
\hline & $\begin{array}{l}\text { Total detection } \\
\text { (number of species) }\end{array}$ & $(\%)$ & $\begin{array}{l}\text { Total detection } \\
\text { (number of species) }\end{array}$ & $(\%)$ \\
\hline Insectivore & 7453 (23 species) & 21.13 & 426 (9 species) & 1.21 \\
\hline Frugivore/Insectivore & 5691 (7 species) & 16.13 & 371 (4 species) & 1.05 \\
\hline Granivore & 4499 (7 species) & 12.76 & 226 (3 species) & 0.64 \\
\hline Omnivore & 3956 (7 species) & 11.22 & 304 (6 species) & 0.86 \\
\hline Piscivore/Carnivore/Insectivore & 3698 (14 species) & 10.48 & 76 (2 species) & 0.22 \\
\hline Granivore/Insectivore & 2372 (4 species) & 6.72 & 284 (4 species) & 0.81 \\
\hline Nectarivore/Insectivore & 2355 (9 species) & 6.67 & 104 (2 species) & 0.29 \\
\hline Frugivore & 1756 (2 species) & 4.98 & 0 & 0.00 \\
\hline Insectivore/Nectarivore/Frugivore & 452 (7 species) & 1.28 & 0 & 0.00 \\
\hline Piscivore/Insectivore & 450 (2 species) & 1.28 & 0 & 0.00 \\
\hline Carnivore/Insectivore & 440 (7 species) & 1.25 & 17 (one species) & 0.05 \\
\hline Piscivore/Carnivore & 224 (one species) & 0.64 & 0 & 0.00 \\
\hline Carnivore & 68 (3 species) & 0.19 & 0 & 0.00 \\
\hline Piscivore & 50 (one species) & 0.14 & 0 & 0.00 \\
\hline Total & 33,464 (94 species) & & 1808 (31 species) & \\
\hline Grand Total $=$ & 35, 272 (98 Species) & & & \\
\hline
\end{tabular}

Table 6. Comparison of bird feeding guilds based on number of observation and number of species in man-made marsh and lake area

\begin{tabular}{lc}
\hline Habitat name & Mean feeding guild \\
\hline Based on number of observation & $2390.3 \mathrm{a}$ \\
Man-made marsh & $129.14 \mathrm{~b}$ \\
Lake area & $6.17 \mathrm{a}$ \\
Based on number of species & $2.21 \mathrm{~b}$ \\
Man-made marsh & \\
Lake area &
\end{tabular}

In contrast, Piscivore $(0.14 \%)$ was the rarest guild comprised of only one species in man-made marsh habitat. However, six feeding guilds i.e., Frugivore, Insectivore/Nectarivore/-Frugivore, Piscivore/Insectivore, Piscivore/Carnivore, Carnivore and Piscivore were absent in the lake area (Table 5).

Significant difference of feeding guilds based on number of bird observation and number of bird species of man-made marsh and lake area was examined through ANOVA and Tukey's (HSD) test. Results revealed that bird feeding guilds of man-made marsh based on number of observation $\left(\mathrm{F}_{1,26}=13.4, \mathrm{p}<0.05\right)$ and based on number of species $\left(\mathrm{F}_{1,26}=7.13, \mathrm{p}<0.05\right)$ were significantly different from lake area (Table 6).

\section{DISCUSSION}

Monitoring the existing bird species diversity is an important step to describe the avian community structures in order to determine whether the habitats for the birds are suitable. The information obtained can be used for effective conservation and successful management (DeSante et al., 2005).

The man-made marsh is dominated with a wide range of water-loving plants including i.e., Eleocharis variegation (Scirpus Variegatus), E. dulcis (Chinese Water Chestnut), Scleria sp. (Nutrush), Eriocaulon longifolium (Pipewort), Nymphaea spp. (Water Lilies), Fimbristylis globulosa (Globe Fimbry), F. miliacea (Grass-like Fimbristylis), Eichhornia crassipes (Water 
Hyacinth) Ludwigia adscendens (Creeping Water Primrose), Philydrum lanuginosum (Wooly Frogs Mouth), Glyceria maxima (Giant Sweet Grass) Phragmites australis (Common Reed), Typha spp. (Cattails), Scirpus spp. (Bulrushes); fruiting trees such as Fragraea fragrans (Heritage Tree), Ficus fistulosa (Common Yellow Stem Fig), F. benjamina (Weeping Fig), Caryota mitis (Fish-tail Palm), Eugenia spp. (Jambu), Garcinia sp. (Asam Gelugor) Dillenia suffruticosa (Simpoh Air or Shrubby Dillenia) and ornamental plants namely Delonix regia (Flame Tree) and Ixora javanica (Jungle Flame). Some parts have been modified to provide nesting and roosting habitat for egrets, herons, egrets and storks. In addition, the lake is devoid of aquatic vegetation and contains trees and shrubs only along the edges.

Diversity of herbaceous emergent vegetation has represented a unique complex ecosystem which had significantly changed the landscape, enhanced the biodiversity, hydrology and natural processes that had attracted diverse wildlife species. From the study, the recording of 98 bird species seems to be the most obvious and significant component of man-made marsh and lake areas that had allowed them to perform multiple activities such as foraging, breeding, perching, nesting and roosting. The functions, values and attributes of this man-made marsh and lake areas had benefitted bird communities and compensated to a certain extent the loss of natural wetlands (Zamora-Orozco et al., 2007; Carmona et al., 2011; Rajpar and Zakaria, 2013).

It was observed that the bird species in the marsh and lake areas were extremely diverse in term of relative abundance, feeding guilds and habitat preference. This indicated that both marsh and lake areas have good ecological conditions that offer diverse resources for a wide array of bird species. Bird species often selected the available habitats in different ways depending on their behaviour. The occurring of water, rich food resources (i.e., amphibians, reptiles, small mammals, aquatic as well as terrestrial invertebrates and fishes) and diverse vegetation types had provided ideal habitat for a wide array of water and terrestrial bird individuals. It was observed that avian community structures in the marsh and lake areas were influenced by a number of variables such as water depth, vegetation structure and composition, food resources and foraging behaviour (Chimney and Gawlik, 2007; Michel et al., 2010; Crampton et al., 2011; Carrascal et al., 2012). It was also found that adjacent dry land area dominated by patches of shrubs and fruiting trees had also attracted a number of bird species particularly upland birds i.e., may be only facultative marsh inhabitants (Knight et al., 2001). It has been reported that birds often select habitats that offer specific foraging and nesting sites (Shochat et al., 2005; Kloskowski et al., 2010; Mcnew et al., 2012).

Higher avian species diversity and richness was recorded in man-made marsh than lake area. This could be due to the presence of suitable foraging, safe nesting and chick rearing sites (i.e., islands, shrub and bamboo patches, dense and scattered trees and reedbeds) and diverse fruiting and flowering tree, grass, reed, shrub, herb and aquatic vegetation species that provides heterogeneous habitats.

Availability of food is probably the most crucial factor that influenced the habitat selection and reproductive success. Foraging behaviour is an essential aspect of avian species in which they obtained and consumed their food sources through using a variety of tactics. It is one of the most important characteristics of the avian species, being necessary for their survival and reproduction. The recording of fourteen feeding guilds in the marsh and lake areas indicated that these areas are highly attractive to many avian species.

Finding a suitable prey is a challenge to avian species. For example; Herons, egrets, bitterns and storks, the most common group of birds, foraged in shallow waters to catch a diversity of aquatic animals such as fishes, amphibians and aquatic invertebrates. They preferred open shallow areas due to richness of food resources and excellent view of approaching predators such water monitor lizards and otters. They avoided the dense vegetated area because it interferes with their movement and foraging efficiency (White, 2003; Lantz et al., 2011). When water level increased, they moved to another area, where water was shallower than their length of tarsus, because, in shallow water prey was easier to catch as compared to deep water (Liordos, 2010; Lantz et al., 2011). Likewise, swamphen and moorhens, preferred to utilize patches of emergent vegetation along the water edges which provided major habitat for different fish and aquatic invertebrates. Their distribution is influenced mainly by the richness and diversity of prey and suitable foraging sites and vegetation cover. In addition, water level also play a significant role on waterbird distribution and diversity. The highest waterbirds species richness occurs in the reedbed where water level is shallow (Holm and Clausen, 2006; Kloskowski et al., 2010; Rajpar and Zakaria, 2011b).

Sandpipers and lapwings probed in soft substrates of ditches, mud and wetland edges to feed on aquatic invertebrates. In soft substrate invertebrates are more vulnerable to forage and easy to catch. In addition, pigeons, starlings and bulbuls were often observed foraging on berries and fruits of shrub plants adjacent to 
the wetland areas. The vegetation adjacent to wetland is an important factor for avian species in determining foraging site selection and also influences the ability of birds to detect approaching predators (White, 2003; Campell, 2008). Robins, pipits, mynas and sparrows selected open grassy lawn to forage on wide array of invertebrates while doves, quails, munias and weavers feed on grass seeds. Furthermore, the results clearly indicated that habitat selection of bird species may vary from species to species, depending upon avian behaviours such as foraging, nesting, loafing and roosting. Finally, many non habitat-related phenomena also influence the habitat selection in birds such as nest predation (Shochat et al., 2005; Marzluff et al., 2007), competition for foraging and nesting (Sherry, 2006), intraspecific attraction (Forsman et al., 2002; Rajchard, 2007; Sridhar et al., 2009) and food limitation (Musil, 2006).

\section{CONCLUSION}

This study highlighted that artificial wetland habitats i.e., man-made marsh and lake are suitable habitats for a diverse avian species to perform various activities such as foraging, breeding, perching, nesting and roosting. Results also showed that man-made marsh is more capable to attract a higher number of bird species and diversity than the lake habitat.

\section{REFERENCES}

Aynalem, S. and A. Bekele, 2008. Species composition, relative abundance and distribution of bird fauna of riverine and wetland habitats of Infranz and Yiganda at southern tip of Lake Tana, Ethiopia. Tropical Ecol., 49: 199-209.

BLI, 2013. Birds are very useful indicators for other kinds of biodiversity. BirdLife International.

Buckland, S.T., D.R. Anderson, K.P. Burnhan, J.L. Lake and J.L. Borchers et al., 2004. Advance Distance Sampling; Estimating Abundance of Biological Populations. 1st Edn, Campman and Hall, London.

Campell, M.O., 2008. The impact of vegetation, river and urban features on waterbird ecology in Glasgow, Scotland. J. Coastal Res., 24: 239-245. DOI: $10.2112 / 07-0835.1$

Carmona, R., V. Ayala-Pérez and A.G. Aguilar, 2011. New and noteworthy waterfowl records at artificial wetlands from Baja California Sur, Mexico. Revista Mexicana de Bio., 82: 721-726.
Carrascal, L.M., J. Seone and S. Villen-Perez, 2012. Temperature and food constraints in wintering birdsan experimental approach in montane Mediterranean oakwoods. Community Ecol., 13: 221-229. DOI: 10.1556/ComEc.13.2012.2.12

Chimney, M.J. and D.E. Gawlik, 2007. The avifauna of constructed treatment wetland in South Florida used for Everglades's restoration. Florida Field Naturalist, 31: 9-21.

Coleman, J.M., O.K. Huh and D.J.R. Braud, 2008. Wetland loss in world deltas. J. Coastal Res., 24: 114. DOI: $10.2112 / 05-0607.1$

Connor, K.J. and S. Gabor, 2006. Breeding waterbird wetland habitat availability and response to waterlevel management in Saint John River floodplain wetlands, New Brunswick. Hydrobiologia, 567: 169-181. DOI: 10.1007/s10750-006-0051-1

Crampton, L.H., W.S. Longland, D.D. Murphy and J.S. Sedinger, 2011. Food abundance determines distribution and density of a frugivorous bird across seasons. Okios, 120: 65-76. DOI: 10.1111/j.16000706.2010.18624.x

DeSante, D.F., M.P. Nott and D.R. Kaschube, 2005. Monitoring, Modeling And Management: Why Base Avian Monitoring on Vital Rates And How Should It Be Done? In: Bird Conservation Implementation and Integration in the Americas, Ralph, C.J. and T.D. Rich (Eds.), Illustrated, pp: 795-804.

Ekert, P., 2009. Shortland Wetlands; Site Management Plan. The Wetlands Centre Australia.

Forsman, J.T., J.T. Seppanen and M. Monkkonen, 2002. Positive fitness consequence of interspecific interaction with a potential on European farmlands. Basic Applic. Ecol., 11: 97-105.

Fraser, L.H. and P.A. Keddy, 2005. The world's Largest Wetlands: Ecology and Conservation. Cambridge University Press, New York, ISBN-10: 052183404X, pp: 488.

Haber, E., 2011. Swamp, marsh and bog. Canadian Encyclopedia.

Henderson, P.A. and R.M.H. Seaby, 2007. Community analysis package 4.0. Pisces Conservation Ltd, Lymington, UK.

Holm, T.E. and P. Clausen, 2006. Effects of water level management on autumn staging waterbird and macrophyte diversity in three Danish coastal lagoons. Biodiversity Conserv., 15: 4399-4423. DOI: $10.1007 / \mathrm{s} 10531-005-4384-2$ 
Ismail, A., F. Rahman and S.Z. Zulkifli, 2012. Status, composition and diversity of avifauna in the artificial Purtajaya wetland and comparison with its two neighboring habitats. Tropical Nat. History, 12: 137-145.

Kadlec, R., R. Knight, J. Vymazal, H. Brix and R. Haberl et al., 2000. Constructed Wetlands for Pollution Control,' Processes, Performance, Design and Operation. 1st Edn., IWA Publishing, London, ISBN-10: 9781900222051, pp: 156.

Kirby, A., 2002. Wastewater treatment using constructed wetlands. Canad. Water Res. J., 27: 263-272. DOI: 10.4296/cwrj2703263

Kloskowski, J., M. Nieoczym, M. Polak and P. Pitucha, 2010. Habitat selection by breeding waterbirds at ponds with size-structured fish populations. Naturwissenschaften. 97: 673-682. DOI: 10,1007/s00114-010-0684-9

Knight, R.L., JR.R.A. Clarke and R.K. Bastian, 2001. Surface Flow (SF) treatment wetlands as habitat for wildlife and humans. Water Sci. Technol., 44: 2737. PMID: 11804106

Lantz, S.M., D.E. Gawlik and M.I. Cook, 2011. The effects of water depth and emergent vegetation on foraging success and habitat selection of wading birds in the Everglades. Waterbirds, 34: 439-447. DOI: 10.1675/063.034.0406

Larsen, S., A. Sorace and L. Mancini, 2010. Riparian bird communities as indicators of human impacts along Mediterranean streams. Environ. Manage., 45: 261-273. DOI: 10.1007/s00267-009-9419-0

Li, H. and S.L. Yang, 2009. Trapping effect of tidal marsh vegetation on suspended sediments, Yangtze Delta. J. Coastal Res., 25: 915-924. DOI: 10.2112/08-1010.1

Liordos, V., 2010. Foraging guilds of waterbirds wintering in a Mediterranean coastal wetland. Zool. Stud., 49: 311-323.

Loaiza, E., 2008. Effects of Different Vegetation Cover Types on Sediment Deposition. Undergraduate Ecology Research Report. pp. 1-7.

Ma, Z., Y. Cai, B. Li and J. Chen, 2010. Managing wetland habitats for waterbirds: An international perspective. Wetlands, 30: 15-27. DOI: 10.1007/s13157-009-0001-6

Marzluff, J.M., J.C. Withey, K.A. Whittaker, M.D. Oleyar and T.M. Unfried et al., 2007. Consequences of habitat utilization by nest predators and breeding songbirds across multiple scales in an urbanizing landscape. Condor, 109: 516-534.
McGraw-Hill, C., 2008. Statistix 8.1. 1st Edn, Analytical Software, Tallahassee, Florida, ISBN-10: 0073402818, pp: 300.

Mcnew, L.B., A.J. Gregory and B.K. Sandercock, 2012. Spatial heterogeneity in habitat selection: Nest site selection by Greater prairie-Chickens. J. Wildlife Manage., 9999: 1-11. DOI: 10.1002/jwmg.493

Michel, P., K.J.M. Dickinson, B.I.P. Barratt and I.G. Jamieson, 2010. Habitat selection in reintroduced bird populations: A case study of Stewart Island robins and South Island saddlebacks on Ulva Island. New Zealand J. Ecol., 34: 237-246.

Mitsch, W.J., 2010. Wetland utilization in the world, protecting sustainable use. The Ohio State University.

Musil, P., 2006. A Review of the Effects of Intensive Fish Production on Waterbird Breeding Population. In: Waterbirds Around the World, Boere, G.C., C.A. Galbaraith and D.A. Stroud (Eds.), The Stationary Office, Edinburg, UK. pp: 520-521.

Nadeau, C.P., C.J. Conway, B.S. Smith and T.E. Lewis, 2008. Maximizing detection probability of wetlanddependent birds during point-count surveys in northwestern Florida. Wilson J. Ornithol., 120: 513518. DOI: 10.1676/07-041.1

Noble, D., M. Everard and A. Joys, 2008. Development of Wild Bird Indicators for Freshwater Wetlands and Waterways: Provisional Indicators. 1st Edn., British Trust for Ornithology, Thetford, ISBN-10: 1906204357, pp: 41.

Rajchard, J., 2007. Interaspecific and interspecific chemsignals in birds: A reviw. Veterinarni Medicina, 52: 385-391.

Rajpar, M.N. and M. Zakaria, 2013. Assessing an artificial wetland in Putrajaya, Malaysia, as an alternate habitat for waterbirds. Waterbirds, 36: 482493. DOI: $10.1675 / 063.036 .0405$

Rajpar, M.N. and M. Zakaria, 2011a. Bird species abundance and their correlationship with microclimate and habitat variables at natural wetland reserve, Peninsular Malaysia. Int. J. Zool. DOI: $10.1155 / 2011 / 758573$

Rajpar, M.N. and M. Zakaria, 2011b. Effects of water level fluctuation on waterbirds distribution and aquatic vegetation composition at natural wetland reserve, Peninsular Malaysia. Int. Scholarly Res. Netw. ISRN Ecol., 2011: 324038-324038. DOI: $10.5440 / 2011 / 324038$ 
Ramírez, F., J. Navarro, I. Afán, K.A. Hobson and A. Delgado et al., 2012. Adapting to a changing world: Unraveling the role of Man-Made habitats as alternative feeding areas for Slender-Billed Gull (Chroicocephalus genei). PLoS ONE, 7: e47551e47551. DOI: 10.1371/journal.pone.0047551

Sherry, D.A., 2006. Foraging ecology of wintering wading birds along the Gulf of Mexico coast. Ph.D Thesis, Texas A and M University, USA.

Shochat, E., M.A. Patten, D.W. Morris, D.L. Reinking, D.H. Wolfe and S.K. Sherrod, 2005. Ecological traps in isodars: Effects of tallgrass prairie management on bird nest success. Oikos, 111: 159169. DOI: 10.1111/j.0030-1299.2005.13907.x

Sim, C.H., 2003. The use of Constructed Wetlands for Wastewater Treatment. 1st Edn., Wetlands Int. Malaysia, pp: 24.

Sim, C.H., M.K. Yusoff, B. Shutes, S.C. Ho and M. Mansor, 2008. Nutrient removal in a pilot and full scale constructed wetland, Putrajaya City, Malaysia. J. Environ. Manage., 88: 307-317. PMID: 17467147

Sridhar, H., G. Beauchamp and K. Shanker, 2009. Why do birds participate in mixed-species foraging flocks? A large-scale synthesis. Animal Behav., 78: 337-347. DOI: 10.1016/j.anbehav.2009.05.008
Vymazal, J., 2010. Constructed wetlands for wastewater treatment. Water, 2: 530-549. DOI: $10.3390 / \mathrm{w} 2030530$

White, C.L., 2003. Habitat value of created wetlands to waterbirds in golf course landscapes. M.Sc Thesis, University of Florida, USA.

Yaukey, P., 2011. Avian use of Bucktown artificial wetland with special emphasis on the population density and reproduction success of the Red-winged Blackbird. Lake Pontchartrain Basin Foundation Report.

Zakaria, M. and M.N. Rajpar, 2013. Density and diversity of waterbirds and terrestrial birds in manmade marsh, Malaysia. Sains Malaysiana, 42: 14831492.

Zakaria, M., M.N. Rajpar and S.A. Sajap, 2009. Species diversity and feeding guilds of birds in Paya Indah Wetland Reserve, Peninsular Malaysia. Int. J. Zool. Res., 5: 86-100. DOI: 10.3923/ijzr.2009.86.100

Zamora-Orozco, E.M., R. Carmona and G. Brabata, 2007. Distribución de aves acuáticas en las lagunas de oxidación de la ciudad de La Paz, Baja California Sur, México. Revista de Biol. Tropical, 55: 617-626. 